\section{P-129 PREVALENCE OF POST-TRAUMATIC STRESS DISORDER IN HEALTH CARE WORKERS FOLLOWING COVID-19: PRELIMINARY RESULTS OF A TWO-GROUP CROSS- SECTIONAL STUDY.}

'Samia Machghoul, Marouen Hayouni, Ines Rassas, Manel Makhloufi, Imen Jammeli, Neila Chaari, Adnene Hanchi, Mohamed Akrout, Irtyah Merchaoui. ${ }^{1}$ Faculty of Medicine of Monastir, Tunisia

\subsection{6/OEM-2021-EPI.207}

Introduction The COVID-19 pandemic has attracted worldwide attention for its rapid and exponential diffusion. The history of past human coronavirus outbreaks resulting in similar health emergencies suggests there will be post-traumatic stress disorders (PTSD) among COVID-19 survivors. This might add to the increasing stress that health care professionals are enduring.

Objectives The purpose of this study was to assess the prevalence and the determinant factors of post-traumatic stress disorder (PTSD) among health care workers with COVID-19.

Methods A two-group cross-sectional study among health care workers (150 cases of COVID-19 and 150 randomly sampled matched controls) was conducted. We present the preliminary results of 91 cases of COVID-19. Demographic data, occupational information and some psychological dimensions were collected using a self-administered questionnaire. Post-traumatic stress disorder was evaluated using the Post-traumatic Stress Disorder Checklist based on the fifth edition of the Diagnostic and Statistical Manual of Mental Disorders.

Results The average age of the population was $36.5 \pm 9.35$ years, with a female predominance and a sex ratio of 0.21 . The most represented professional categories were nurses and medical residents. The acute clinical manifestations lasted between 10 to 20 days in $41 \%$ of the patients. Moderate to severe levels of anxiety and depression were noted among respectively $27.5 \%$ and $14 \%$ of the population. PTSD was found among $8.8 \%$ of the study population. Significant correlations were found between COVID-19-PTSD scores and caring for children or parents $\left(\mathrm{p}=10^{-3}\right)$, anxiety level $\left(\mathrm{p}=10^{-3}\right)$, being admitted to the hospital $(\mathrm{p}=0.008)$ and the symptoms presented $(\mathrm{p}=0.003)$.

Conclusion These preliminary results are relevant from a clinical point of view because they suggest that the COVID-19 pandemic could be considered as a traumatic event among health care workers. Psychological and organizational interventions to counteract short- and long-term psychopathological effects, consequent to the COVID-19 pandemic, appear to be necessary.

\section{P-131 COVID-19 IN ESSENTIAL ACTIVITIES: REPORT ON SUCCESSFUL MEASURES IN OCCUPATIONAL HEALTH FOR 7,000 WORKERS IN AN ENVIRONMENTAL SANITATION COMPANY IN BRAZIL}

${ }^{1}$ Telma de Cassia dos Santos Nery, Ayrton Jouti, Wellington Yschisaki, Monica Aparecida de Oliveira Augusto. 'HCFMUSP, Brazil

\subsection{6/OEM-2021-EPI.208}

Introduction According to a WHO note, since March 11, 2020, the planet has been living with an important pandemic. Human behavior and the environment are different at this point. There are countless deaths. Workers in environmental sanitation services such as water and sewage are considered essential and need a special approach to COVID-19. Until May 9, 2021, there were in Brazil 15,000,000 infected people. This work describes the safety measures of a group of 7,000 workers of the largest environmental sanitation company in Latin America.

Objective To describe measures adopted to deal with COVID19 through the safety and health protocol.

Methodology We describe the participation of the occupational medical service in the construction and institution of an appropriate protocol from April 2020 to April 2021 considering a bibliographic survey, management discussions, discussions with workers and measures implemented on the site, monitoring of positive cases, guidelines and preventive health care.

Results Approximately 1,000 telephone surveys were carried out for everyone suspected and/or diagnosed COVID-19. The implementation of a health protocol and daily dissemination of guidance via videos/reports. $60 \%$ of workers were allocated to teleworking. 770 cases and 12 deaths were identified. 12\% of cases occurred in workers of essential activities. Such data in this Unit corresponded to $45 \%$ of the cases of the entire company and $30 \%$ of deaths.

Conclusion The adoption of specific measures and protocols, increased the participation of workers with the impact of COVID-19 on workers in environmental sanitation. The adoption of preventive measures and protocols before COVID-19 can contribute to the reduction of cases in essential work activities.

\section{P-132 A PROFILE OF OCCUPATIONAL ACCIDENTS IN BRAZIL ACCORDING OF NOTIFICATION, IN THE PERIOD 2012 TO 2020}

${ }^{1}$ Telma de Cassia dos Santos Nery, Daniel Nery Cardoso. ${ }^{1}$ HCFMUSP, Brazil

\subsection{6/OEM-2021-EPI.209}

Introduction Occupational Accidents (OA) in Brazil are mandatory to report, but they are very underreported. In 2020, Brazil had a population of 212 million inhabitants. According to the International Labor Organization, Brazil occupies the fourth position in the world ranking of occupational accidents, behind China, India and Indonesia. There are various notification systems and an important underreporting. In 2012, according to a survey by the IBGE, 5 million suffered OA. Officially registered data for 2019 were 700,000 cases. Analyzing existing data considering notification systems can contribute to the identification of profiles and the adoption of specific measures that involve communities beyond specific ones.

Objective To analyze OA in Brazil in the period 2012 to 2020.

Methodology Descriptive study based on the analysis of data from the Health and Safety Observatory at Work, from 2012 to 2020. Data: by state, economic sectors, most frequent injuries, most frequently affected body parts, groups of causative agents, occupations, social security absences, gender.

Results In 2020 there were 400 thousand OA. 2013 was the year with the highest number of deaths 2,841. The state of SP was the most prevalent $(35 \%)$ of OA , the Hospital economic sector represented $10 \%$ of all accidents, 72 thousand received social security benefits. It is estimated that $25 \%$ of $\mathrm{OA}$ in 2018 were underreported. The most frequent injuries (21\%) being cuts, lacerations. The most affected part was the 